\title{
Representation of Auditory Motion Directions and Sound Source Locations in the Human Planum Temporale
}

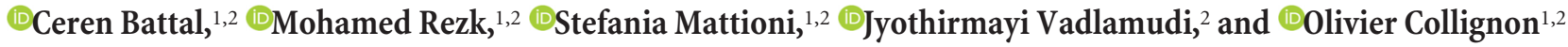 \\ ${ }^{1}$ Crossmodal Perception and Plasticity Laboratory, Center of Mind/Brain Sciences, University of Trento, I-38122 Trento, Italy, and ${ }^{2}$ Crossmodal Perception \\ and Plasticity Laboratory, Institute for Research in Psychology, Institute of Neuroscience, Université catholique de Louvain, 1348 Louvain, Belgium
}

The ability to compute the location and direction of sounds is a crucial perceptual skill to efficiently interact with dynamic environments. How the human brain implements spatial hearing is, however, poorly understood. In our study, we used fMRI to characterize the brain activity of male and female humans listening to sounds moving left, right, up, and down as well as static sounds. Whole-brain univariate results contrasting moving and static sounds varying in their location revealed a robust functional preference for auditory motion in bilateral human planum temporale (hPT). Using independently localized hPT, we show that this region contains information about auditory motion directions and, to a lesser extent, sound source locations. Moreover, hPT showed an axis of motion organization reminiscent of the functional organization of the middle-temporal cortex (hMT +/V5) for vision. Importantly, whereas motion direction and location rely on partially shared pattern geometries in hPT, as demonstrated by successful cross-condition decoding, the responses elicited by static and moving sounds were, however, significantly distinct. Altogether, our results demonstrate that the hPT codes for auditory motion and location but that the underlying neural computation linked to motion processing is more reliable and partially distinct from the one supporting sound source location.

Key words: auditory motion; direction selectivity; fMRI; multivariate analyses; planum temporale; spatial hearing

Significance Statement

Compared with what we know about visual motion, little is known about how the brain implements spatial hearing. Our study reveals that motion directions and sound source locations can be reliably decoded in the human planum temporale (hPT) and that they rely on partially shared pattern geometries. Our study, therefore, sheds important new light on how computing the location or direction of sounds is implemented in the human auditory cortex by showing that those two computations rely on partially shared neural codes. Furthermore, our results show that the neural representation of moving sounds in hPT follows a "preferred axis of motion" organization, reminiscent of the coding mechanisms typically observed in the occipital middle-temporal cortex (hMT+/V5) region for computing visual motion.

\section{Introduction}

While the brain mechanisms underlying the processing of visual localization and visual motion have received considerable attention (Newsome and Paré, 1988; Movshon and Newsome, 1996;

\footnotetext{
Received Sept. 8, 2018; revised Dec. 20, 2018; accepted Dec. 21, 2018.

Author contributions: C.B., M.R., and 0.C. designed research; C.B. performed research; C.B., M.R., S.M., and J.V. contributed unpublished reagents/analytic tools; C.B. analyzed data under the supervision of $0 . C$.; C.B. and O.C. wrote the paper core of the paper, all authors commented and approved the final version of the paper.

The project was funded by the European Research Council starting Grant MADVIS (Project 337573 - MADVIS), awarded to 0.C.. M.R. and J.V. are PhD fellows and 0.C. is a research associate at the Fond National de la Recherche Scientifique de Belgique. We thank Giorgia Bertonati, Marco Barilari, Stefania Benetti, Valeria Occelli, and Stephanie Cattoir, who helped with the data acquisition; Jorge Jovicich, who helped with setting up the fMRI acquisition parameters; and Pietro Chiesa, who provided continuing support for the auditory hardware.

The authors declare no competing financial interests.

Correspondence should be addressed to Ceren Battal at ceren.batta@@uclouvain.be or Olivier Collignon at olivier.collignon@uclouvain.be.

https://doi.org/10.1523/JNEUROSCI.2289-18.2018

Copyright $\odot 2019$ the authors $\quad 0270-6474 / 19 / 392208-13 \$ 15.00 / 0$
}

Braddick et al., 2001), much less is known about how the brain implements spatial hearing. The representation of auditory space relies on the computations and comparison of intensity, temporal and spectral cues that arise at each ear (Searle et al., 1976; Blauert, 1982). In the auditory pathway, these cues are both processed and integrated in the brainstem, thalamus, and cortex to create an integrated neural representation of auditory space (Boudreau and Tsuchitani, 1968; Goldberg and Brown, 1969; Knudsen and Konishi, 1978; Ingham et al., 2001; for review, see Grothe et al., 2010).

Similar to the dual-stream model in vision (Ungerleider and Mishkin, 1982; Goodale and Milner, 1992), partially distinct ventral "what" and dorsal "where" auditory processing streams have been proposed in audition (Romanski et al., 1999; Rauschecker and Tian, 2000; Recanzone, 2000; Tian et al., 2001; Warren and Griffiths, 2003; Barrett and Hall, 2006; Lomber and Malhotra, 2008; Collignon et al., 2011). In particular, the dorsal stream is 


\section{A Recording moving and static sounds}

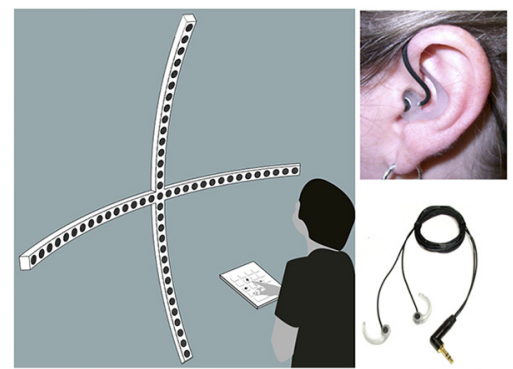

\section{B fMRI design}

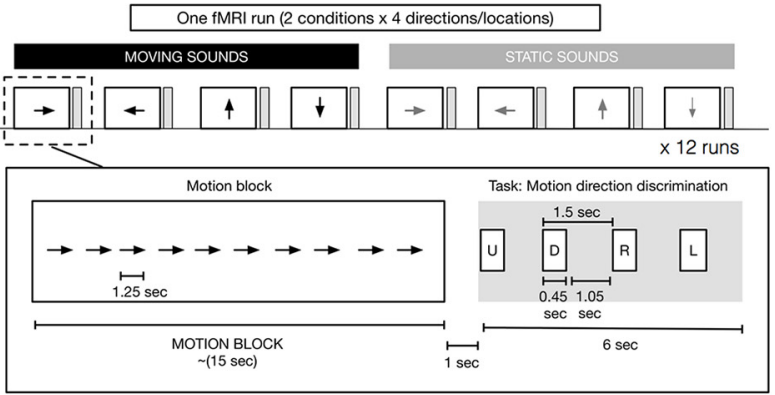

C Behavioral results inside fMRI

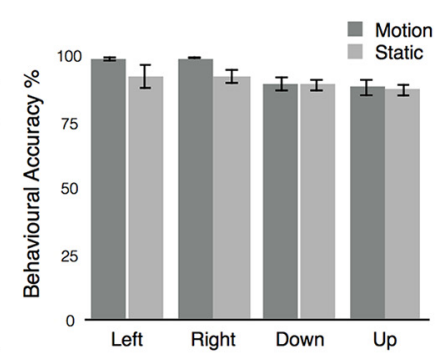

Figure 1. Stimuli and experimental design. $A$, The acoustic apparatus used to present auditory moving and static sounds while binaural recordings were performed for each participant before the fMRI session. $\boldsymbol{B}$, Auditory stimuli presented inside the MRI scanner consisted of the following eight conditions: leftward, rightward, upward and downward moving stimuli; and left, right, up, and down static stimuli. Each condition was presented for $15 \mathrm{~s}$ ( 12 repetitions of $1250 \mathrm{~ms}$ sound, no ISI), and followed by a $7 \mathrm{~s}$ gap for indicating the corresponding direction/location in space and $8 \mathrm{~s}$ of silence (total interblock interval was $15 \mathrm{~s}$ ). Sound presentation and response button press were pseudorandomized. Participants were asked to respond as accurately as possible during the gap period. C, The behavioral performance inside the scanner.

thought to process sound source location and motion both in animals and humans (Rauschecker and Tian, 2000; Alain et al., 2001; Maeder et al., 2001; Tian et al., 2001; Arnott et al., 2004). However, it remains poorly understood whether the human brain implements the processing of auditory motion and location using distinct, similar, or partially shared neural substrates (Zatorre et al., 2002; Smith et al., 2010; Poirier et al., 2017).

One candidate region that might integrate spatial cues to compute motion and location information is the human planum temporale (hPT; Baumgart et al., 1999; Warren et al., 2002; Barrett and Hall, 2006). hPT is located in the superior temporal gyrus, posterior to Heschl's gyrus, and is typically considered part of the dorsal auditory stream (Rauschecker and Tian, 2000; Warren et al., 2002; Derey et al., 2016; Poirier et al., 2017). Some authors have suggested that hPT engages in both the processing of moving sounds and the location of static sound sources ( $\mathrm{Za}$ torre et al., 2002; Smith et al., 2004, 2007, 2010; Krumbholz et al., 2005; Barrett and Hall, 2006; Derey et al., 2016). This proposition is supported by early animal electrophysiological studies demonstrating the neurons in the auditory cortex that are selective to sound source location and motion directions (Altman, 1968, 1994; Benson et al., 1981; Middlebrooks and Pettigrew, 1981; Imig et al., 1990; Rajan et al., 1990; Poirier et al., 1997; Doan et al., 1999). In contrast, other studies in animals (Poirier et al., 2017) and humans (Baumgart et al., 1999; Lewis et al., 2000; Bremmer et al., 2001; Pavani et al., 2002; Hall and Moore, 2003; Krumbholz et al., 2005; Poirier et al., 2005) pointed toward a more specific role of hPT for auditory motion processing.

The hPT, selective to process auditory motion/location in a dorsal "where" pathway, is reminiscent of the human middle temporal cortex (hMT+/V5) in the visual system. hMT+/V5 is dedicated to process visual motion (Watson et al., 1993; Tootell et al., 1995; Movshon and Newsome, 1996) and displays a columnar organization tuned to axis of motion direction (Albright et al., 1984; Zimmermann et al., 2011). However, whether hPT discloses similar characteristic tuning properties remains unknown.

The main goals of the present study were threefold. First, using multivariate pattern analysis (MVPA), we investigated whether information about auditory motion direction and sound source location can be retrieved from the pattern of activity in hPT. Further, we asked whether the spatial distribution of the neural representation is in the format of "preferred axis of motion/location" as observed in the visual motion selective regions
(Albright et al., 1984; Zimmermann et al., 2011). Finally, we aimed at characterizing whether the processing of motion direction (e.g., going to the left) and sound source location (e.g., being in the left) rely on partially common neural representations in the hPT.

\section{Materials and Methods Participants}

Eighteen participants with no reported auditory problems were recruited for the study. Two participants were excluded due to poor spatial hearing performance in the task, as it was lower by $>2.5$ SDs than the average of the participants. The final sample included 16 right-handed participants ( 8 females; age range, $20-42$ years; mean \pm SD age, $32 \pm 5.7$ years). Participants were blindfolded and instructed to keep their eyes closed throughout the experiments and practice runs. All the procedures were approved by the research ethics boards of the Centre for Mind/Brain Sciences and the University of Trento. Experiments were undertaken with the understanding and written consent of each participant.

\section{Auditory stimuli}

Our limited knowledge of the auditory space processing in the human brain might be a consequence of the technical challenge of evoking a vivid perceptual experience of auditory space while using neuroimaging tools such as fMRI, EEG, or MEG. In this experiment, to create an externalized ecological sensation of sound location and motion inside the MRI scanner, we relied on individual in-ear stereo recordings that were recorded in a semianechoic room and on 30 loudspeakers on horizontal and vertical planes, mounted on two semicircular wooden structures with a radius of $1.1 \mathrm{~m}$ (Fig. 1A). Participants were seated in the center of the apparatus with their head on a chinrest, such that the speakers on the horizontal and vertical planes were equally distant from participants' ears. Then, these recordings were replayed to the participants when they were inside the MRI scanner. By using such a sound system with in-ear recordings, auditory stimuli automatically convolved with each individuals' own pinna and head-related transfer function to produce a salient auditory perception in external space.

The auditory stimuli were prepared using custom MATLAB scripts (r2013b; MathWorks). Auditory stimuli were recorded using binaural in-ear omnidirectional microphones ("flat" frequency range, 20-20,000 $\mathrm{Hz}$; model TFB-2, Sound Professionals) connected to a portable Zoom $\mathrm{H} 4 \mathrm{n}$ digital wave recorder (16 bit, stereo, $44.1 \mathrm{kHz}$ sampling rate). Microphones were positioned at the opening of the participant's left and right auditory ear canals. While auditory stimuli were played, participants were listening without performing any task with their head fixed to the chinrest in front of them. Binaural in-ear recordings allowed combining binaural properties such as interaural time and intensity differ- 
ences, and participant-specific monaural filtering cues to create reliable and ecological auditory space sensation (Pavani et al., 2002).

\section{Stimuli recording}

Sound stimuli consisted of $1250 \mathrm{~ms}$ pink noise (50 ms rise/fall time). In the motion condition, the pink noise was presented moving in the following four directions: leftward, rightward, upward, and downward. Moving stimuli covered $120^{\circ}$ of the space/visual field in horizontal and vertical axes. To create the perception of smooth motion, the $1250 \mathrm{~ms}$ of pink noise was fragmented into 15 pieces of equal length with each 83.333 ms fragment being played every two speakers, and moved one speaker at a time, from outer left to outer right (rightward motion), or vice versa for the leftward motion. For example, for the rightward sweep, sound was played through speakers located at $-60^{\circ}$ and $-52^{\circ}$ consecutively, followed by $-44^{\circ}$, and so on. A similar design was used for the vertical axis. This resulted in participants perceiving moving sweeps covering an arc of $120^{\circ}$ in $1250 \mathrm{~ms}$ (speed, $96^{\circ} \mathrm{s}$; fade in/out, $50 \mathrm{~ms}$ ) containing the same sounds for all four directions. The choice of the movement speed of the motion stimuli aimed to create a listening experience relevant to conditions of everyday life. Moreover, at such velocity it has been demonstrated that human listeners are not able to make the differences between concatenated static stimuli from motion stimuli elicited by a single moving object (Poirier et al., 2017), supporting the participant's report that our stimuli were perceived as smoothly moving (no perception of successive snapshots). In the static condition, the same pink noise was presented separately at one of the following four locations: left, right, up, and down. Static sounds were presented at the second-most outer speakers $\left(-56^{\circ}\right.$ and $+56^{\circ}$ in the horizontal axis, and $+56^{\circ}$ and $-56^{\circ}$ in the vertical axis) to avoid possible reverberation differences at the outermost speakers. The static sounds were fixed at one location at a time instead of presented in multiple locations (Krumbholz et al., 2005; Smith et al., 2004, 2010; Poirier et al., 2017). This strategy was purposely adopted for two main reasons. First, randomly presented static sounds can evoke a robust sensation of auditory apparent motion (Strybel and Neale, 1994; Lakatos and Shepard, 1997; for review, see Carlile and Leung, 2016). Second, and crucially for the purpose of the present experiment, presenting static sounds located in a given position and moving sounds directed toward the same position allowed us to investigate whether moving and static sounds share a common representational format using crosscondition classification (see below), which would have been impossible if the static sounds were randomly moving in space.

Before the recordings, the sound pressure levels (SPLs) were measured from the participant's head position and ensured that each speaker conveys a $65 \mathrm{~dB}$-A SPL. All participants reported a strong sensation of auditory motion and were able to detect locations with high accuracy (Fig. 1C). Throughout the experiment, participants were blindfolded. Stimuli recordings were conducted in a session that lasted $\sim 10 \mathrm{~min}$, requiring the participant to remain still during this period.

\section{Auditory experiment}

Auditory stimuli were presented via MR-compatible closed-ear headphones (500-10 KHz frequency response; Serene Sound, Resonance Technology) that provided average ambient noise cancellation of $\sim 30$ dB-A. Sound amplitude was adjusted according to each participant's comfort level. To familiarize the participants with the task, they completed a practice session outside of the scanner until they reached $>80 \%$ accuracy.

Each run consisted of the eight auditory categories (four motion and four static) randomly presented using a block design. Each category of sound was presented for $15 \mathrm{~s}$ [ 12 repetitions of $1250 \mathrm{~ms}$ sound, no interstimulus interval (ISI)] and followed by $7 \mathrm{~s}$ gap for indicating the corresponding direction/location in space and $8 \mathrm{~s}$ of silence (total interblock interval, $15 \mathrm{~s}$ ). The ramp (50 ms fade in/out) applied at the beginning and at the end of each sound creates static bursts and minimized adaptation to the static sounds. During the response gap, participants heard a voice saying "left," "right," "up," and "down" in pseudorandomized order. Participants were asked to press a button with their right index finger when the direction or location of the auditory block was matching with the auditory cue (Fig. 1B). The number of targets and the order (positions 1-4) of the correct button press were balanced across category. This procedure was adopted to ensure that the participants gave their response using equal motor command for each category and to ensure the response is produced after the end of the stimulation period for each category. Participants were instructed to emphasize the accuracy of response but not the reaction times.

Each scan consisted of one block of each category, resulting in a total of eight blocks per run, with each run lasting 4 min and $10 \mathrm{~s}$. Participants completed a total of 12 runs. The order of the blocks was randomized within each run and across participants.

Based on pilot experiments, we decided to not rely on a sparsesampling design, as is sometimes done in the auditory literature to present the sounds without the scanner background noise (Hall et al., 1999). These pilot experiments showed that the increase in the signal-to-noise ratio potentially provided by sparse sampling did not compensate for the loss in the number of volume acquisitions. Indeed, pilot recordings on participants not included in the current sample showed that, given a similar acquisition time between sparse-sampling designs (several options tested) and continuous acquisition, the activity maps elicited by our spatial sounds contained higher and more reliable $\beta$ values using continuous acquisition.

\section{fMRI data acquisition and analyses}

\section{Imaging parameters}

Functional and structural data were acquired with a $4 \mathrm{~T}$ Bruker MedSpec Biospin MR scanner, equipped with an eight-channel head coil. Functional images were acquired with $\mathrm{T} 2{ }^{*}$-weighted gradient echoplanar sequence. Acquisition parameters were as follows: repetition time (TR), $2500 \mathrm{~ms}$; echo time (TE), $26 \mathrm{~ms}$; flip angle (FA), 73º field of view, 192 $\mathrm{mm}$; matrix size, $64 \times 64$; and voxel size, $3 \times 3 \times 3 \mathrm{~mm}^{3}$. A total of 39 slices was acquired in an ascending feet-to-head interleaved order with no gap. The three initial scans of each acquisition run were discarded to allow for steady-state magnetization. Before every two EPI runs, we performed an additional scan to measure the point-spread function of the acquired sequence, including fat saturation, which served for distortion correction that is expected with high-field imaging (Zeng and Constable, 2002).

A high-resolution anatomical scan was acquired for each participant using a T1-weighted 3D MP-RAGE sequence (176 sagittal slices; voxel size, $1 \times 1 \times 1 \mathrm{~mm}^{3}$; field of view, $256 \times 224 \mathrm{~mm}$; TR, $2700 \mathrm{~ms}$; TE, 4.18 ms; FA, $7^{\circ}$; inversion time, $1020 \mathrm{~ms}$ ). Participants were blindfolded and instructed to lie still during acquisition, and foam padding was used to minimize scanner noise and head movement.

\section{Univariate fMRI analysis: whole brain}

Raw functional images were preprocessed and analyzed with SPM8 [Welcome Trust Centre for Neuroimaging London, UK (https://www.fil. ion.ucl.ac.uk/spm/software/spm8/)] implemented in MATLAB R2014b (MathWorks). Before the statistical analysis, our preprocessing steps included slice time correction with reference to the middle temporal slice, realignment of functional time series, the coregistration of functional and anatomical data, spatial normalization to an EPI template conforming to the Montreal Neurological Institute (MNI) space, and spatial smoothing (Gaussian kernel, 6 mm FWHM).

To obtain blood oxygenation level-dependent activity related to auditory spatial processing, we computed single-subject statistical comparisons with a fixed-effect general linear model (GLM). In the GLM, we used eight regressors from each category (four motion direction, four sound source location). The canonical double-gamma hemodynamic response function implemented in SPM8 was convolved with a box-car function to model the above-mentioned regressors. Motion parameters derived from realignment of the functional volumes (three translational motion and three rotational motion parameters), button press, and the four auditory response cue events were modeled as regressors of no interest. During the model estimation, the data were high-pass filtered with a cutoff $128 \mathrm{~s}$ to remove the slow drifts/low-frequency fluctuations from the time series. To account for serial correlation due to noise in fMRI signal, autoregression [AR (1)] was used. 
To obtain activity related to auditory processing in the whole brain, the contrasts tested the main effect of each category ([Left Motion], [Right Motion], [Up Motion], [Down Motion], [Left Static], [Right Static], [Up Static], [Down Static]). To find brain regions responding preferentially to the moving and static sounds, we combined all motion conditions [Motion] and all static categories [Static]. The contrasts tested the main effect of each condition ([Motion], [Static]), and comparison between the conditions ([Motion > Static], and [Static > Motion]). These linear contrasts generated statistical parametric maps (SPM[T]), which were further spatially smoothed (Gaussian kernel, $8 \mathrm{~mm}$ FWHM) and entered in a second-level analysis, corresponding to a random-effects model, accounting for intersubject variance. One-sample $t$ tests were run to characterize the main effect of each condition ([Motion], [Static]) and the main effect of motion processing ([Motion $>$ Static]) and static location processing ([Static $>$ Motion]). Statistical inferences were performed at a threshold of $p<0.05$ corrected for multiple comparisons [familywise error (FWE) corrected] either over the entire brain volume or after correction for multiple comparisons over small spherical volumes (12 mm radius) located in regions of interest (ROIs). Significant clusters were anatomically labeled using the xjView Matlab toolbox (http://www.alivelearn.net/xjview) or structural neuroanatomy information provided in the Anatomy Toolbox (Eickhoff et al., 2007).

\section{ROI analyses}

ROI definition. Due to the hypothesis-driven nature of our study, we defined $\mathrm{hPT}$ as an a priori ROI for statistical comparisons and to define the volume in which we performed multivariate pattern classification analyses.

To avoid any form of double dipping that may arise when defining the ROI based on our own data, we decided to independently define hPT, using a meta-analysis method of quantitative association test, implemented via the on-line tool Neurosynth (Yarkoni et al., 2011) using the term query "Planum Temporale." Rather than showing which regions are disproportionately reported by studies where a certain term is dominant [uniformity test; $\mathrm{P}$ (activation $\mid$ term)], this method identifies regions whose report in a neuroimaging study is diagnostic of a certain term being dominant in the study [association test; $\mathrm{P}$ (term $\mid$ activation)]. As such, the definition of this ROI was based on a set of 85 neuroimaging studies at the moment of the query (September 2017). This method provides an independent method to obtain masks for further ROI analysis. The peak coordinate from the meta-analysis map was used to create $6 \mathrm{~mm}$ spheres (117 voxels) around the peak $z$-values of hPT [peak MNI coordinates $(-56,-28,8]$ and $[60,-28,8]$; left hPT (lhPT) and right hPT (rhPT) hereafter].

Additionally, hPT regions of interest were individually defined using anatomical parcellation with FreeSurfer (http://surfer.nmr.mgh. harvard.edu). The individual anatomical scan was used to perform cortical anatomical segmentation according to the atlas by Destrieux et al. (2010). We selected a planum temporale label defined as [G_temp_supPlan_tempo, 36] bilaterally. We equated the size of the ROI across participants and across hemispheres to 110 voxels (each voxel being $3 \mathrm{~mm}$ isotropic). For anatomically defined hPT ROIs, all further analyses were performed in subject space for enhanced anatomical precision and to avoid spatial normalization across participants. We replicated our pattern of results in anatomically defined parcels (lhPT and rhPT) obtained from the single-subject brain segmentation (for further analysis, see Battal et al., 2018).

Univariate. The $\beta$ parameter estimates of the four motion directions and four sound source locations were extracted from lhPT and rhPT regions (Fig. 2C). To investigate the presence of motion directions/sound source location selectivity and condition effect in hPT regions, we performed a 2 Conditions (hereafter referring to the stimuli type: motion, static) $\times 4$ Orientations (hereafter referring to either the direction or location of the stimuli: left, right, down, and up) repeated-measures ANOVA in each hemisphere separately on these $\beta$ parameter estimates. Statistical results were then corrected for multiple comparisons (number of ROIs $\times$ number of tests) using the false discovery rate (FDR) method (Benjamini and Yekutieli, 2001). A Greenhouse-Geisser correction was

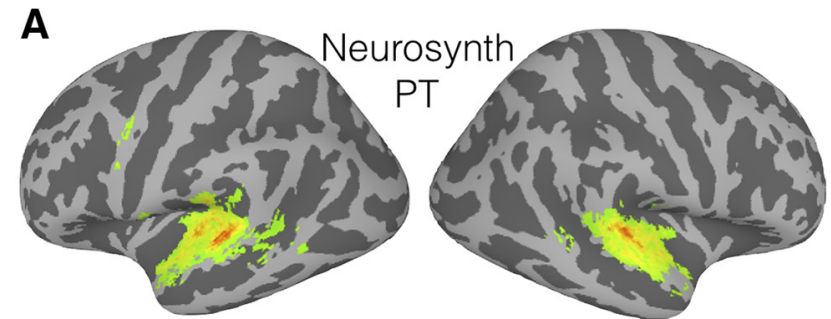

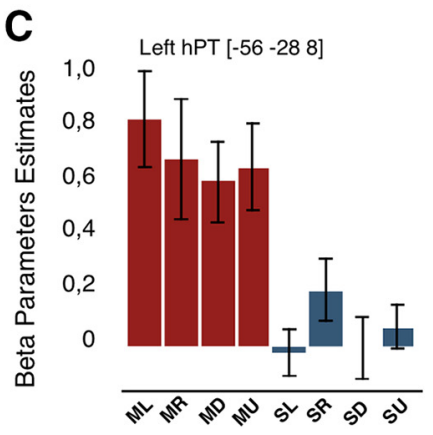

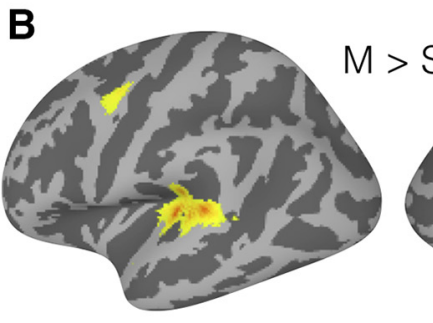

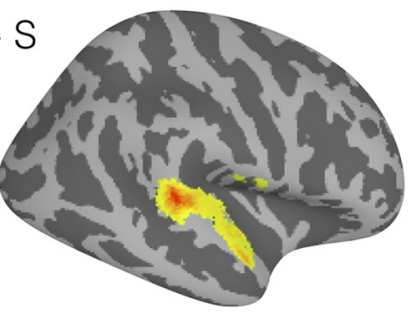

10 Right hPT [60 -28 8]

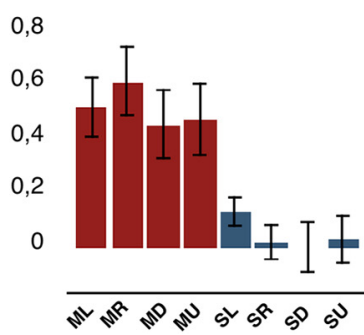

Figure 2. Univariate whole-brain results. $A$, Association test map was obtained from the on-line tool Neurosynth using the term Planum Temporale (FDR corrected, $p<0.05$ ). The black spheres are illustrations of a drawn mask (radius $=6 \mathrm{~mm}, 117$ voxels) around the peak coordinate from Neurosynth (search term Planum Temporale, meta-analysis of 85 studies). $\boldsymbol{B}$, Auditory motion processing [Motion $>$ Static] thresholded at $p<0.05$, whole-brin FWE corrected. C, Mean activity estimates (arbitrary units \pm SEM) associated with the perception of auditory motion direction (red) and sound source location (blue). ML, Motion left; MR, motion right; MD, motion down; MU, motion up; SL, static left; SR, static right; SD, static down; and SU, static up.

applied to the degrees of freedom and significance levels whenever an assumption of sphericity was violated.

\section{ROI multivariate pattern analyses}

Within-condition classification. Four-class and binary classification analyses were conducted within the hPT region to investigate the presence of auditory motion direction and sound source location information in this area. To perform multivoxel pattern classification, we used a univariate one-way ANOVA to select a subset of voxels $(n=110)$ that are showing the most significant signal variation between the categories of stimuli (in our study, between orientations). This feature selection not only ensures a similar number of voxels within a given region across participants (dimensionality reduction), but, more importantly, identifies and selects voxels that are carrying the most relevant information across categories of stimuli (Cox and Savoy, 2003; De Martino et al., 2008), therefore minimizing the chance to include in our analyses voxels carrying noises unrelated to our categories of stimuli.

MVPAs were performed in the lhPT and rhPT. Preprocessing steps were identical to the steps performed for univariate analyses, except for functional time series that were smoothed with a Gaussian kernel of 2 mm (FWHM). MVPA was performed using CoSMoMVPA (http:// www.cosmomvpa.org/; Oosterhof et al., 2016), implemented in MATLAB. Classification analyses were performed using support vector machine (SVM) classifiers as implemented in LIBSVM (https://www. csie.ntu.edu.tw/ cjlin/libsvm/; Chang and Lin, 2011). A general linear model was implemented in SPM8, where each block was defined as a 
regressor of interest. A $\beta$ map was calculated for each block separately. Two multiclass and six binary linear SVM classifiers with a linear kernel with a fixed regularization parameter of $\mathrm{C}=1$ were trained and tested for each participant separately. The two multiclass classifiers were trained and tested to discriminate among the response patterns of the four auditory motion directions and locations, respectively. Four binary classifiers were used to discriminate brain activity patterns for motion and location within axes (left vs right motion, left vs right static, up vs down motion, up vs down static; hereafter called within-axis classification). We used eight additional classifiers to discriminate across axes (left vs up, left vs down, right vs up, and right vs down motion directions; left vs up, left vs down, right vs up, and right vs down sound source locations; hereafter called across-axes classification).

For each participant, the classifier was trained using a cross-validation leave-one-out procedure where training was performed with $n-1$ runs, and testing was then applied to the remaining one run. In each crossvalidation fold, the $\beta$ maps in the training set were normalized ( $z$-scored) across conditions, and the estimated parameters were applied to the test set. To evaluate the performance of the classifier and its generalization across all of the data, the previous step was repeated 12 times, where in each fold a different run was used as the testing data and the classifier was trained on the other 11 runs. For each region per participant, a single classification accuracy was obtained by averaging the accuracies of all cross-validation folds.

Cross-condition classification. To test whether motion directions and sound source locations share a similar neural representation in hPT region, we performed cross-condition classification. We performed the same steps as for the within-condition classification as described above but trained the classifier on sound source locations and tested on motion directions, and vice versa. The accuracies from the two cross-condition classification analyses were averaged. For interpretability reasons, crosscondition classification was only interpreted on the stimuli categories that the classifiers discriminated reliably (above chance level) for both motion and static conditions (e.g., if discrimination of left vs right was not successful in one condition, either static or motion, then the left vs right cross-condition classification analysis was not performed).

Within-orientation classification. To foreshadow our results, crosscondition classification analyses (see previous section) showed that motion directions and sound source locations share, at least partially, a similar neural representation in hPT region. To further investigate the similarities/differences between the neural patterns evoked by motion directions and sound source locations in the hPT, we performed four binary classifications in which the classifiers were trained and tested on the same orientation pairs: leftward motion versus left static, rightward motion versus right static, upward motion versus up static, and downward motion versus down static. If the same orientation (leftward and left location) across conditions (motion and static) generates similar patterns of activity in hPT region, the classifier would not be able to differentiate leftward motion direction from left sound location. However, significant within-orientation classification would indicate that the evoked patterns within hPT contain differential information for motion direction and sound source location in the same orientation (e.g., left).

The mean of the four binary classifications was computed to produce one accuracy score per ROI. Before performing the within-orientation and cross-condition MVPA, each individual pattern was normalized separately across voxels so that any cross-orientation or within-orientation classification could not be due to global univariate activation differences across the conditions.

\section{Statistical analysis: MVPA}

Statistical significance in the multivariate classification analyses was assessed using nonparametric tests permuting condition labels and bootstrapping (Stelzer et al., 2013). Each permutation step included shuffling of the condition labels and rerunning the classification 100 times on the single-subject level. Next, we applied a bootstrapping procedure to obtain a group-level null distribution that is representative of the whole group. From each individual's null distribution, one value was randomly chosen and averaged across all of the participants. This step was repeated 100,000 times, resulting in a group-level null distribution of 100,000 values. The classification accuracies across participants was considered as significant if $p<0.05$ after corrections for multiple comparisons (number of ROIs $\times$ number of tests) using the FDR method (Benjamini and Yekutieli, 2001).

A similar approach was adopted to assess statistical difference between the classification accuracies of two auditory categories (e.g., four motion direction vs four sound source location, left motion vs left static, left motion vs up motion). We performed additional permutation tests (100,000 iterations) by building a null distribution for $t$ statistics after randomly shuffling the classification accuracy values across two auditory categories and recalculating the two-tailed $t$ test between the classification accuracies of these two categories. All $p$ values were corrected for multiple comparisons (number of ROIs $X$ number of tests) using the FDR method (Benjamini and Yekutieli, 2001).

\section{Representation similarity analysis}

Neural dissimilarity matrices

To further explore the differences in the representational format between sound source locations and motion directions in hPT region, we relied on representation similarity analysis (RSA; Kriegeskorte et al., 2008). More specifically, we tested the correlation between the representational dissimilarity matrix (RDM) of rhPT and lhPT in each participant with different computational models that included condition-invariant models assuming orientation invariance across conditions (motion, static), condition-distinct models assuming that sound source location and motion direction sounds elicit highly dissimilar activity patterns, and a series of intermediate graded models between them. The RSA was performed using the CosmoMVPA toolbox (Oosterhof et al., 2016) implemented in MATLAB. To perform this analysis, we first extracted in each participant the activity patterns associated with each condition (Edelman et al., 1998; Haxby et al., 2001). Then, we averaged individual-subject statistical maps (i.e., activity patterns) to have a mean pattern of activity for each auditory category across runs. Finally, we used Pearson's linear correlation as the similarity measure to compare each possible pair of the activity patterns evoked by the four different motion directions and four different sound source locations. This resulted in an $8 \times 8$ correlation matrix for each participant that was then converted into an RDM by computing $1-$ correlation. Each element of the RDM contains the dissimilarity index between the patterns of activity generated by two categories, in other words the RDM represents how different the neural representation of each category is from the neural representations of all the other categories in the selected ROI. The 16 neural RDMs ( 1 per participant) for each of the two ROIs were used as neural input for RSA.

\section{Computational models}

To investigate shared representations between auditory motion directions and sound source locations, we created multiple computational models ranging from a fully condition-distinct model to a fully condition-invariant model with intermediate gradients in between (Zabicki et al., 2017; see Fig. 4C).

Condition-distinct model. The condition-distinct models assume that dissimilarities between motion and static condition is 1 (i.e., highly dissimilar), meaning that neural responses/patterns generated by motion and static conditions are totally unrelated. For instance, there would be no similarity between any motion directions with any sound source location. The dissimilarity values in the diagonal were set to 0 , simply reflecting that neural responses for the same direction/location are identical to themselves.

Condition-invariant model. The condition-invariant models assume a fully shared representation for specific/corresponding static and motion conditions. For example, the models consider the neural representation for the left sound source location and the left motion direction highly similar. All within-condition (left, right, up, and down) comparisons are set to 0 (i.e., highly similar), regardless of their auditory condition. The dissimilarities between different directions/locations are set to 1, meaning that each within-condition sound (motion or static) is different from all the other within-condition sounds.

Intermediate models. To detect the degree of similarity/shared representation between motion direction and sound source location patterns, 
we additionally tested two classes of five different intermediate models. The two classes were used to deepen the understanding of the characteristic tuning of hPT for separate direction/location or axis of motion/ location. The two model classes represent two different possibilities. The first scenario was labeled as Within-Axis Distinct, and these models assume that each of the four directions/locations (i.e., left, right, up, and down) would generate a distinctive neural representation different from all of the other within-condition sounds (e.g., the patterns of activity produced by the left category are highly different from the patterns produced by right, up, and down categories; see Fig. $4 C$, top). To foreshadow our results, we observed a preference for axis of motion in MVP classification; therefore, we created another class of models to further investigate neural representations of within-axis and across-axes of auditory motion/space. The second scenario was labeled with Within-Axis Combined, and these models assume that opposite direction/locations within the same axis would generate similar patterns of activity [e.g., the pattern of activity of horizontal (left and right) categories are different from the patterns of activity of vertical categories; up and down; see Fig. 4C, bottom].

In all intermediate models, the values corresponding to the dissimilarity between same auditory spaces (e.g., left motion and left location) were gradually modified from 0.9 (motion and static conditions are mostly distinct) to 0.1 (motion and static conditions mostly similar). These models were labeled IM9, IM7, IM5, IM3, and IM1, respectively.

In all condition-distinct and intermediate models, the dissimilarity of within-condition sounds was fixed to 0.5 , and the dissimilarity of withinorientation sounds was fixed to 1 . Across all models, the diagonal values were set to 0 .

\section{Performing RSA}

We computed Pearson's correlation to compare neural RDMs and computational model RDMs. The resulting correlation captures which computational model better explains the neural dissimilarity patterns between motion direction and sound source location conditions. To compare computational models, we performed the Mann-WhitneyWilcoxon rank-sum test between for every pair of models. All $p$ values were then corrected for multiple comparisons using the FDR method (Benjamini and Yekutieli, 2001). To test differences between two classes of models (Within-Axis Combined vs Within-Axis Distinct), within each class, the correlation coefficient values were averaged across hemispheres and across models. Next, we performed permutation tests (100,000 iterations) by building a null distribution for differences between classes of models after randomly shuffling the correlation coefficient values across two classes, and recalculating the subtraction between the correlation coefficients of Within-Axis Combined and Within-Axis Distinct classes.

To visualize the distance between the patterns of the motion directions and sound source locations, we used multidimensional scaling (MDS) to project the high-dimensional RDM space onto two dimensions with the neural RDMs that were obtained from both $\mathrm{lhPT}$ and rhPT.

Additionally, the single-subject $8 \times 8$ correlation matrices were used to calculate the reliability of the data considering the signal-to-noise ratio of the data (Kriegeskorte et al., 2007). For each participant and each ROI, the RDM was correlated with the averaged RDM of the rest of the group. The correlation values were then averaged across participants. This provided the maximum correlation that can be expected from the data.

\section{Results}

\section{Behavioral results}

During the experiment, we collected target direction/location discrimination responses (Fig. 1C). The overall accuracy scores were entered into $2 \times 4$ (Condition, Orientation) repeatedmeasures ANOVA. No main effect of Condition $\left(F_{(1,15)}=2.22\right.$; $p=0.16$ ) was observed, indicating that the overall accuracy while detecting the direction of motion or sound source location did not differ. There was a significant main effect of orientation $\left(F_{(1.6,23.7)}=11.688 ; p=0.001\right)$, caused by greater accuracy in the horizontal orientations (left and right) compared with the vertical orientations (up and down). Post hoc two-tailed $t$ tests
Table 1. Results of the univariate analyses for the main effect of auditory motion processing [motion $>$ static]

\begin{tabular}{lrrrrrr}
\hline & \multicolumn{7}{c}{ MNI coordinates (mm) } \\
\cline { 3 - 4 } Area & $K$ & $x$ & $y$ & $z$ & $Z$ & $p$ \\
\hline Motion > Static & & & & & & \\
$\quad$ L planum temporale & 10,506 & -46 & -32 & 10 & 6.63 & $0.000^{*}$ \\
L Middle temporal G & & -56 & -38 & 14 & 6.10 & $0.000^{*}$ \\
L Precentral G & & -46 & -4 & 52 & 5.25 & $0.004^{*}$ \\
L Putamen & & -22 & 2 & 2 & 4.98 & $0.011^{*}$ \\
L Middle temporal G & 43 & -50 & -52 & 8 & 3.79 & $0.01^{\#}$ \\
R Superior temporal G & 7074 & 66 & -36 & 12 & 6.44 & $0.000^{*}$ \\
R Superior temporal G & & 62 & -2 & -4 & 5.73 & $0.000^{*}$ \\
R Superior temporal G & & 52 & -14 & 0 & 5.56 & $0.001^{*}$ \\
R Precentral G & & 50 & 2 & 50 & 4.70 & $0.032^{*}$ \\
R Superior frontal S & 159 & 46 & 0 & 50 & 4.40 & $0.001^{\#}$ \\
R Middle temporal G & 136 & 42 & -60 & 6 & 4.31 & $0.001^{\#}$ \\
R Middle occipital G & 24 & 44 & -62 & 6 & 3.97 & $0.006^{\#}$ \\
\hline
\end{tabular}

Coordinates reported in this table are significant ( $p<0.05$, FWE corrected) after correction over small spherical volumes (SVCs, $12 \mathrm{~mm}$ radius) of interest (\#) or over the whole brain $(*)$. The coordinates used for correction over small SVCs are as follows (in $x, y, z$, in MNI space): left middle temporal gyrus (hMT+/V5), $[-42,-64,4]$ (Dormal et al., 2016); right middle temporal gyrus (hMT +/V5) $[42,-60,4]$ (Dormal et al., 2016); right superior frontal sulcus $[32,0,48]$ (Collignon et al., 2011); right middle occipital gyrus $[48,-76,6]$ (Collignon et al., 2011). $K$, Number of voxels when displayed at $p$ (uncorrected) $<0.001 ; L$, left; $R$, right; $G$, gyrus; $S$, sulcus.

(Bonferroni corrected) revealed that accuracies did not reveal significant differences within horizontal orientations (left vs right; $t_{(15)}=-0.15, p=1$ ), and within vertical orientations (up vs down; $\left.t_{(15)}=0.89, p=1\right)$. However, left orientation accuracy was greater compared with down $\left(t_{(15)}=3.613, p=0.005\right)$ and up $\left(t_{(15)}=4.51, p<0.001\right)$ orientation accuracies, and right orientation accuracy was greater compared with the down $\left(t_{(15)}=3.76\right.$, $p=0.003)$ and up $\left(t_{(15)}=4.66, p<0.001\right)$ orientation accuracies. No interaction between Condition and Orientation was observed, pointing out that differences between orientations in terms of performance expresses both for static and motion. A Greenhouse-Geisser correction was applied to the degrees of freedom and significance levels whenever an assumption of sphericity was violated.

\section{fMRI results}

Whole-brain univariate analyses

To identify brain regions that are preferentially recruited for auditory motion processing, we performed a univariate random effects-GLM contrast (Motion > Static; Fig. 2B). Consistent with previous studies (Pavani et al., 2002; Warren et al., 2002; Poirier et al., 2005; Getzmann and Lewald, 2012; Dormal et al., 2016), whole-brain univariate analysis revealed activation in the superior temporal gyri, bilateral hPT, precentral gyri, and anterior portion of middle temporal gyrus in both hemispheres (Fig. 2B, Table 1). The most robust activation (resisting whole-brain FWE correction, $p<0.05$ ) was observed in the bilateral hPT (peak MNI coordinates $[-46,-32,10]$ and $[60,-32,12])$. We also observed significant activation in occipitotemporal regions (in the vicinity of anterior hMT+/V5), as suggested by previous studies (Warren et al., 2002; Poirier et al., 2005; Dormal et al., 2016).

\section{ROI univariate analyses}

Beta parameter estimates were extracted from the predefined ROIs (see Materials and Methods) for the four motion directions and four sound source locations from the auditory experiment (Fig. 2C). We investigated the condition effect and the presence of direction/location selectivity in $\mathrm{lhPT}$ and $\mathrm{rhPT}$ regions separately by performing $2 \times 4$ (Conditions, Orientations) repeated measures of ANOVA with $\beta$ parameter estimates. In lhPT, the 
main effect of Conditions was significant $\left(F_{(1,15)}=37.28, p<\right.$ $0.001)$, indicating that auditory motion evoked a higher response compared with static sounds. There was no significant main effect of Orientations $\left(F_{(1.5,22.5)}=0.77, p=0.44\right)$, and no interaction $\left(F_{(3,45)}=2.21, p=0.11\right)$. Similarly, in rhPT, only the main effect of Conditions was significant $\left(F_{(1,15)}=37.02, p<0.001\right)$. No main effect of Orientation $\left(F_{(1.5,23.2)}=1.43, p=0.26\right)$ or interaction $\left(F_{(3,45)}=1.74, p=0.19\right)$ was observed. Overall, brain activity in the hPT, as measured with a $\beta$ parameter estimate extracted from the univariate analysis, did not provide evidence of motion direction or sound source location selectivity.

\section{ROI multivariate pattern analyses}

To further investigate the presence of information about auditory motion direction and sound source location in hPT, we ran multiclass and binary multivariate pattern classifications. Figure $3 A-C$ shows the mean classification accuracy across categories in each ROI.

Within condition classification. Multiclass across four conditions, the classification accuracy in the hPT was significantly above chance (chance level, 25\%) in both hemispheres for motion direction (lhPT: mean $\pm \mathrm{SD}=38.4 \pm 7, p<0.001$; rhPT: mean $\pm \mathrm{SD}=37.1 \pm 6.5, p<0.001)$ and sound source location (lhPT: mean $\pm \mathrm{SD}=32.4 \pm 6.7, p<0.001$; rhPT: mean $\pm \mathrm{SD}=$ $31.2 \pm 7.5, p<0.001$; Fig. $3 A$ ). In addition, we assessed the differences between classification accuracies for motion and static stimuli by using permutation tests in lhPT $(p=0.024)$ and rhPT $(p=0.024)$, indicating greater accuracies for classifying motion direction than sound source location across all regions.

Binary within-axis classification. Binary horizontal (left vs right) within-axis classification showed significant results in both lhPT and rhPT for static sounds (lhPT: mean $\pm \mathrm{SD}=58.6 \pm$ $14.5, p<0.001$; rhPT: mean $\pm \mathrm{SD}=56.5 \pm 11.9, p=0.008$; Fig. $3 C)$, while motion classification was significant only in the rhPT (mean $\pm \mathrm{SD}=55.5 \pm 13.9, p=0.018$; Fig. $3 B$ ). Moreover, binary vertical (up vs down) within-axis classification was significant only in the lhPT for both motion (mean $\pm \mathrm{SD}=55.7 \pm 7.1, p=$ 0.01 ) and static (mean $\pm \mathrm{SD}=54.9 \pm 11.9, p=0.03$ ) conditions (Fig. $3 B, C$ ).

Binary across-axis classification. We used eight additional binary classifiers to discriminate across axes-moving and static sounds. Binary across-axes (Left vs Up, Left vs Down, Right vs Up, and Right vs Down) classifications were significantly above chance level in the bilateral hPT for motion directions (Left vs Up: lhPT: mean $\pm \mathrm{SD}=65.8 \pm 14.8, p<0.001$; rhPT: mean \pm $\mathrm{SD}=64.8 \pm 9.4, p<0.001$; Left vs Down: lhPT: mean $\pm \mathrm{SD}=$ $74 \pm 15.9, p<0.001$; r PT: mean $\pm \mathrm{SD}=66.9 \pm 10.4, p<0.001$; Right vs Up: lhPT: mean $\pm \mathrm{SD}=72.4 \pm 15.8, p<0.001$; rhPT: mean $\pm \mathrm{SD}=71.4 \pm 13.3, p<0.001$; Right vs Down: lhPT: mean $\pm \mathrm{SD}=73.4 \pm 11.8, p<0.001 ;$ rhPT: mean $\pm \mathrm{SD}=$ $68.2 \pm 15.9, p<0.001$; Fig. $3 B$ ). However, sound source location across-axes classifications were significant only in certain conditions (Left vs Up rhPT: mean $\pm \mathrm{SD}=56 \pm 12.7, p=0.018$; Left vs Down: lhPT: mean $\pm \mathrm{SD}=55.2 \pm 11.2, p=0.024$; rhPT: mean $\pm \mathrm{SD}=57.3 \pm 17.2, p=0.003$; Right vs Down lhPT: mean $\pm \mathrm{SD}=57.6 \pm 8.4, p=0.005$; Fig. $3 C$ ).

"Axis of motion" preference. To test whether neural patterns within hPT contain information about opposite directions/locations within an axis, we performed four binary within-axis classifications. Similar multivariate analyses were performed to investigate the presence of information about across-axes directions/locations. The classification accuracies were plotted in Figure $3, B$ and $C$.

\section{Left hPT}

Right hPT

\section{A MVPA 4 Directions/Locations}
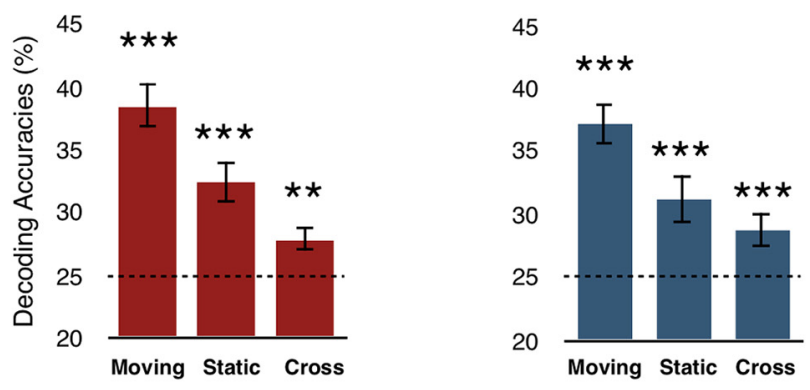

B MVPA Within/Across-Axis Directions
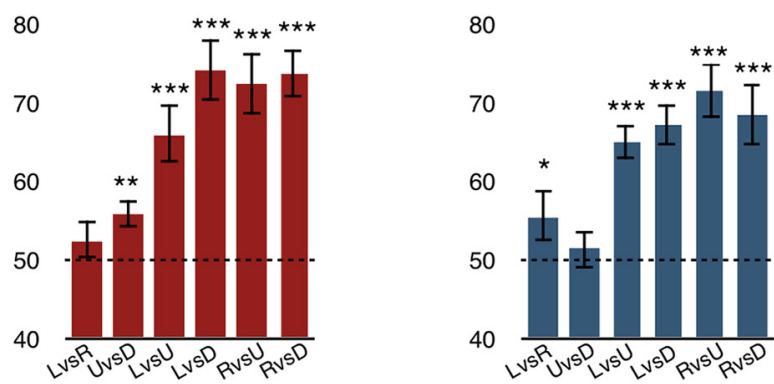

\section{C}

VPA Within/Across-Axis Locations
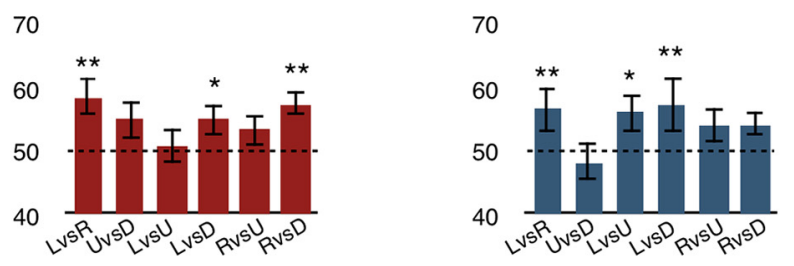

Figure 3. Within-classification and cross-classification results. $\boldsymbol{A}$, Classification results for the four conditions in the functionally defined hPT region. Within-condition and cross-condition classification results are shown in the same bar plots. Moving, four motion directions; Static, four sound source locations; Cross, cross-condition classification accuracies. $\boldsymbol{B}$, Classification results of within-axes (left vs right, up vs down) and across-axes (left vs up, left vs down, right vs up, right vs down) motion directions. C, Classification results of within-axes (left vs right, up vs down) and across-axes (left vs up, left vs down, right vs up, right vs down) sound source locations. LvsR, Left vs Right; UvsD, Up vs Down; LvsU, Left vs Up; LvsD, Left vs Down; RvsU, Right vs Up; RvsD, Right vs Down classifications. FDR-corrected $p$ values: ${ }^{*} p<0.05,{ }^{* *} p<0.01$, ${ }^{* * *} p<0.001$ testing differences against chance level (dotted lines; see Materials and Methods).

In motion direction classifications, to assess the statistical difference between classification accuracies of across axes (left vs up, left vs down, and right vs up, right vs down) and within axes (left vs right and up vs down) directions, we performed pairwise permutation tests that were FDR corrected for multiple comparisons. Across-axes classification accuracies in lhPT ([left vs up] vs [left vs right]: $p=0.006$; [left vs down] vs [left vs right]: $p<$ 0.001; [right vs down] vs [left vs right]: $p<0.001$; [right vs up] vs [left vs right]: $p=0.001$ ), and rhPT ([left vs up] vs [left vs right]: $p=0.029$; [left vs down] vs [left vs right]: $p=0.014$; [right vs down] vs [left vs right]: $p=0.02$; [right vs up] vs [left vs right]: $p=0.003$ ) were significantly higher compared with the horizontal within-axis classification accuracies. Similarly, across-axes classification accuracies were significantly higher when compared with vertical within-axis classification accuracies in lhPT 
([up vs down] vs [left vs up]: $p=0.02$; [up vs down] vs [left vs down]: $p=0.001$; [up vs down] vs [right vs up]: $p=0.001$; [up vs down] vs [right vs down]: $p<0.001$ ) and rhPT ([up vs down] vs [left vs up]: $p=0.001$; [up vs down] vs [left vs down]: $p=$ 0.001; [up vs down] vs [right vs up]: $p=0.001$; [up vs down] vs [right vs down]: $p=0.002$ ). No significant difference was observed between the within-axis classifications in lhPT ([left vs right] vs [up vs down]: $p=0.24$ ) and rhPT ([left vs right] vs [up vs down]: $p=0.31$ ). Similarly, no significance difference was observed among the across-axes classification accuracies in the bilateral hPT.

In static sound location classifications, no significant difference was observed between across-axes and within-axes classification accuracies, indicating that the classifiers did not perform better when discriminating sound source locations across axes compared with the opposite locations.

One may wonder whether the higher classification accuracy for across axes compared with within axes relates to the perceptual differences (e.g., difficulty in localizing) in discriminating sounds within the horizontal and vertical axes. Indeed, because we opted for an ecological design reflecting daily life listening condition, we observed, as expected, that discriminating vertical directions was more difficult than discriminating horizontal ones (Middlebrooks and Green, 1991). To address this issue, we replicated our classification MVPAs after omitting the four participants showing the lowest performance in discriminating the vertical motion direction, leading to comparable performance (at the group level) within and across axes. We replicated our pattern of results by showing preserved higher classification accuracies across axes than within axis. Moreover, while accuracy differences between across-axes and within-axes classifications was observed only in the motion condition, behavioral differences were observed in both static and motion conditions. To assess whether the higher across-axes classification accuracies are due to differences in difficulties between horizontal and vertical sounds, we performed correlation analysis. For each participant, the behavioral performance difference between horizontal (left and right) and vertical (up and down) conditions was calculated. The difference between the classification accuracies within axes (left vs right and up vs down) and across axes (left vs up, left vs down, right vs up, and right vs down) was calculated. Spearman correlation between the $(\Delta)$ behavioral performance and $(\Delta)$ classification accuracies was not significant in the bilateral hPT (lhPT: $r=0.18, p=0.49$; rhPT: $r=0.4, p=0.12$ ). An absence of correlation suggests that there is no relation between the difference in classification accuracies and the perceptual difference. These results strengthen the notion that the axis of motion organization observed in PT does not simply stem from behavioral performance differences.

Cross-condition classification. To investigate whether motion direction and sound source locations rely on shared representation in $\mathrm{hPT}$, we trained the classifier to distinguish neural patterns from the motion directions (e.g., going to the left) and then tested on the patterns elicited by static conditions (e.g., being in the left), and vice versa.

Cross-condition classification revealed significant results across four directions/locations (lhPT: mean $\pm \mathrm{SD}=27.8 \pm 5.3$, $p=0.008$; rhPT: mean $\pm \mathrm{SD}=28.7 \pm 3.8, p<0.001$; Fig. $3 A$ ). Within-axis categories did not reveal any significant crosscondition classification. These results suggest that a partial overlap between the neural patterns of moving and static stimuli in the hPT.
Within orientation classification. Cross-condition classification results indicated a shared representation between motion directions and sound source locations. Previous studies have argued that successful cross-condition classification reflects an abstract representation of stimuli conditions (Hong et al., 2012; Fairhall and Caramazza, 2013; Higgins et al., 2017). To test this hypothesis, patterns of the same orientation of motion and static conditions (e.g., leftward motion and left location) were involved in within-orientation MVPA. The rational was that if the hPT region carries a fully abstract representation of space, withinorientation classification would give results in favor of the null hypothesis (no differences within the same orientation). In the within-orientation classification analysis, accuracies from the four within-orientation classification analyses were averaged and survived FDR corrections in bilateral hPT (lhPT: mean $\pm \mathrm{SD}=$ $65.6 \pm 5, p<0.001$; rhPT: mean $\pm \mathrm{SD}=61.9 \pm 5.6, p<0.001)$, indicating that the neural patterns of motion direction can be reliably differentiated from sound source location within hPT (Fig. 4A).

\section{RSA}

\section{Multidimensional scaling}

Visualization of the representational distance between the neural patterns evoked by motion directions and sound source locations further supported that within-axis directions show similar geometry compared with the across-axes directions, therefore, it is more difficult to differentiate the neural patterns of opposite directions in MVP-classification. MDS also showed that in both lhPT and rhPT, motion directions and sound source locations are separated into 2 clusters (Fig. $4 B$ ).

\section{Correlation with computational models}

The correlation between model predictions and neural RDMs for the lhPT and rhPT is shown in Figure $4 D$. The cross-condition classification results indicated a shared representation within the neural patterns of hPT for motion and static sounds. We examined the correspondence between the response pattern dissimilarities elicited by our stimuli, with 14 different model RDMs that included a fully condition distinct, fully condition-invariant models, and intermediate models with different degrees of shared representation.

The first class of computational RDMs was modeled with the assumption that the neural patterns of within-axis sounds are fully distinct. The analysis revealed a negative correlation with the fully condition-invariant model in the bilateral hPT (lhPT, mean $r \pm \mathrm{SD}=-0.12 \pm 0.18$; rhPT, mean $r \pm \mathrm{SD}=-0.01 \pm 0.2)$ that increased gradually as the models progressed in the conditiondistinct direction. The model that best fit the data was the M9 model in the bilateral hPT (lhPT, mean $r \pm \mathrm{SD}=0.66 \pm 0.3$; rhPT, mean $r \pm \mathrm{SD}=0.65 \pm 0.3$ ). A similar trend was observed for the second class of models that have the assumption of withinaxis sounds that evoke similar neural patterns. The conditioninvariant model provided the least explanation of the data (lhPT, mean $r \pm \mathrm{SD}=0.14 \pm 0.25$; rhPT, mean $r \pm \mathrm{SD}=0.2 \pm 0.29$ ), and correlations gradually increased as the models progressed in the condition-distinct direction. The winner models in this class were the models M9 in lhPT and M7 in the rhPT (lhPT, mean $r \pm$ $\mathrm{SD}=0.67 \pm 0.22 ; \mathrm{rhPT}$, mean $r \pm \mathrm{SD}=0.69 \pm 0.15$ ).

In addition, we assessed differences between correlation coefficients for computational models using the Mann-Whitney-Wilcoxon rank-sum test for each class of models and hemispheres separately (Fig. 4D,E). In the Within-Axis Distinct class in lhPT, pairwise comparisons of correlation coefficients did not show a 
A

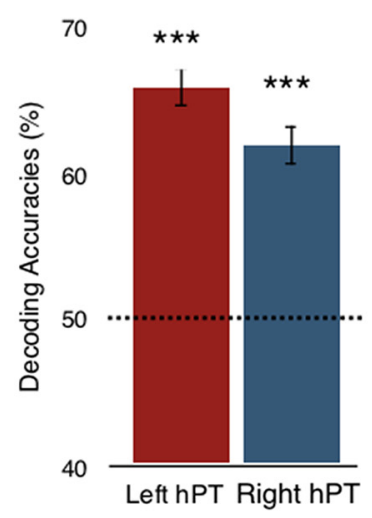

B
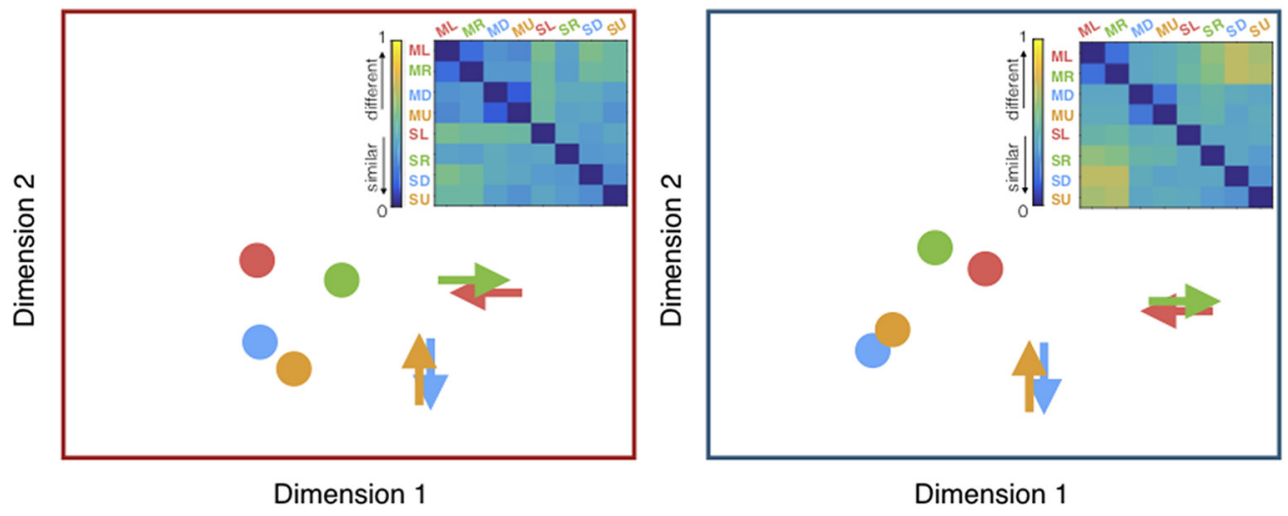

IM.9
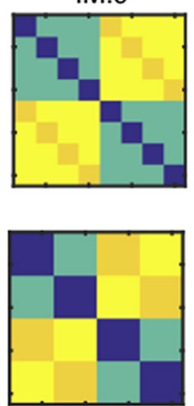

IM.9
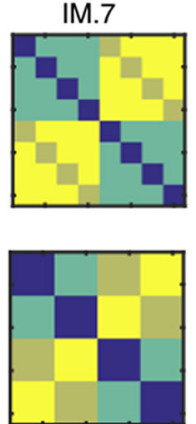

IM.7
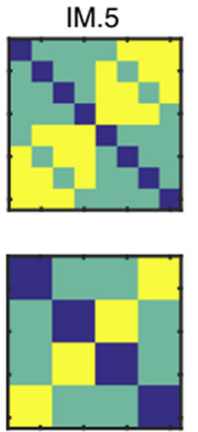

IM.5
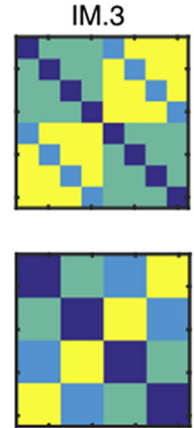

IM.3

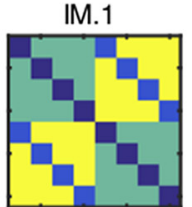

Condition Invariant

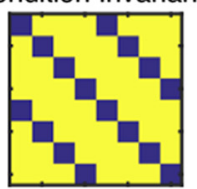

Left hPT

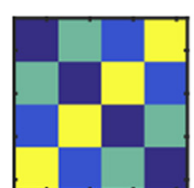

IM.1

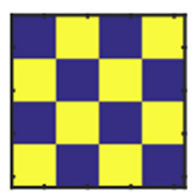

Right hPT

D
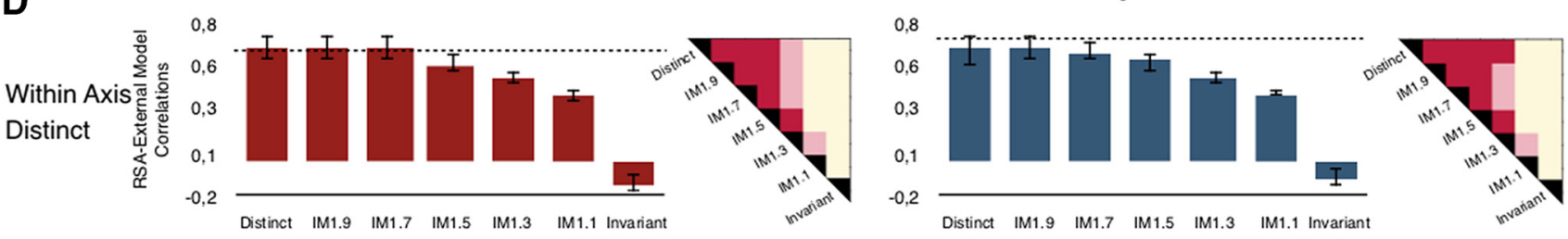

E

Left hPT

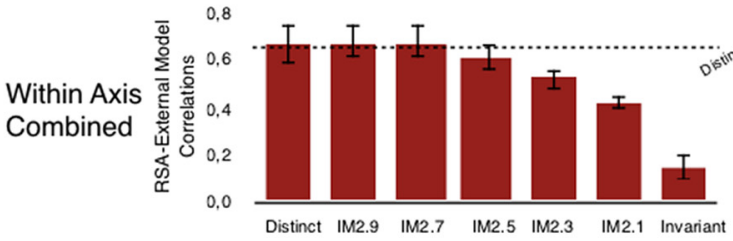

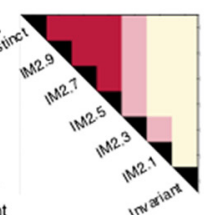

Right hPT

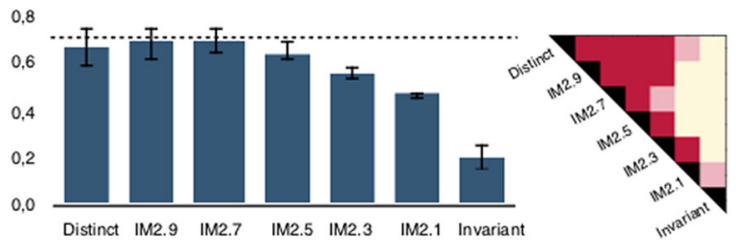

Figure 4. Pattern dissimilarity between motion directions and sound source locations. A, Across-condition classification results across four conditions are represented in each R0I (IhPT and rhPT). Four binary classifications [leftward motion vs left location], [rightward motion vs right location], [upward motion vs up location], and [downward motion vs down location] were computed and averaged to produce one accuracy score per ROI. FDR-corrected $p$ values: ${ }^{* * *} p<0.001$. Dotted lines represent chance level. $\boldsymbol{B}$, The inset shows neural RDMs extracted from IhPT and rhPT, and the MDS plot visualizes the similarities of the neural pattern elicited by four motion directions (arrows) and four sound source locations (dots). Color codes for arrow/dots are as follows: green indicates left direction/location; red indicates right direction/location; orange indicates up direction/location; and blue indicates down direction/location. ML, Motion left; MR, motion right; MD, motion down; MU, motion up; SL, static left; SR, static right; SD, static down; SU, static up. C-E, The results of RSA in hPT are represented. C, RDMs of the computational models that assume different similarities of the neural pattern based on auditory motion and static conditions. $\boldsymbol{D}, \boldsymbol{E}$, RSA results for every model and each ROI. For each ROl, the dotted lines represent the reliability of the data considering the signal-to-noise ratio (see Materials and Methods), which provides an estimate of the highest correlation we can expect in a given ROI when correlating computational models and neural RDMs. Error bars indicate the SEM. IM1, Intermediate models with within-axis conditions distinct; IM2, Intermediate model with within-axis conditions combined. Each right up corner of the bar plots shows visualization of significant differences for each class of models and hemispheres separately (Mann-Whitney-Wilcoxon rank-sum test, FDR corrected).

significant difference for [Condition Distinct versus IM.9 $(p=$ $0.8)$ ], [Condition Distinct versus IM7 $(p=0.6) ; \mathrm{rhPT}]$, and [Condition Distinct versus IM.5 $(p=0.09)$ ]; however, as the models progressed further in the condition-invariant direction, the difference between correlation coefficients for models reached significance ([Condition Distinct vs IM.3], $p=0.012$; [Condition Distinct vs IM.1], $p=0.007$; [Condition Distinct vs Condition Invariant], $p<0.001$ ), indicating that the Condition- 
Distinct model provided a stronger correlation compared with the models representing conditions similarly. In rhPT, the ranksum tests between each pairs revealed no significant difference for [Condition Distinct versus IM.9 $(p=0.9)$ ], [Condition Distinct versus IM7 $(p=0.7)$ ], [Condition Distinct versus IM.5 $(p=$ $0.3)$ ], and also [Condition Distinct versus IM.3 $(p=0.06)$ ]; however, as the models progressed further in the condition-invariant direction, the difference between correlation coefficients for models reached significance ([Condition Distinct vs IM.1], $p=$ 0.006; [Condition Distinct vs Condition Invariant], $p<0.001$ ).

The two classes of models were used to deepen our understanding of the characteristic tuning of hPT for separate direction/location or axis of motion/location. To reveal the differences between Within-Axis Distinct and Within-Axis Combine classes of models, we relied on a two-sided signed-rank test. Within-Axis Combined models explained our stimuli space better than Within-Axis Distinct models supporting similar pattern representation within planes $(p=0.0023)$.

\section{Discussion}

In line with previous studies, our univariate results demonstrate higher activity for moving than for static sounds in the superior temporal gyri, bilateral hPT, precentral gyri, and anterior portion of middle temporal gyrus in both hemispheres (Baumgart et al., 1999; Pavani et al., 2002; Warren et al., 2002; Krumbholz et al., 2005; Poirier et al., 2005). The most robust cluster of activity was observed in the bilateral hPT (Fig. 2B, Table 1). Moreover, activity estimates extracted from independently defined hPT also revealed higher activity for moving relative to static sounds in this region. Both whole-brain and ROI analyses therefore clearly indicated a functional preference (expressed here as higher activity level estimates) for motion processing over sound source location in bilateral hPT regions (Fig. 2).

Does hPT contain information about specific motion directions and sound source locations? At the univariate level, our four (left, right, up, and down) motion directions and sound source locations did not evoke differential univariate activity in hPT region (Fig. 2C). We then performed multivariate pattern classification and observed that bilateral hPT contains reliable distributed information about the four auditory motion directions (Fig. 3 ). Our results are therefore similar to the observations made with $\mathrm{fMRI}$ in the human visual motion area hMT+/V5 showing reliable direction-selective multivariate information despite comparable voxelwise univariate activity levels across directions (Kamitani and Tong, 2006; but see Zimmermann et al., 2011). Within-axis MVP classification results revealed that even if both horizontal (left vs right), and vertical (up vs down) motion directions can be classified in the hPT region (Fig. 3C,D), across-axes (e.g., left vs up) direction classifications revealed higher decoding accuracies compared with within-axis classifications. Such enhanced classification accuracy across axes versus within axis is reminiscent of observations made in $\mathrm{MT}+/ \mathrm{V} 5$, where a largescale axis of motion-selective organization was observed in nonhuman primates (Albright et al., 1984) and in area hMT+/V5 in humans (Zimmermann et al., 2011). Further examination with RSA provided additional evidence that within-axis combined models (aggregating the opposite directions/location) better explain the neural representational space of hPT by showing higher correlation values compared with within-axis distinct models (Fig. $4 D, E$ ). Our findings suggest that the topographic organization principle of hMT $+/ \mathrm{V} 5$ and hPT shows similarities in representing motion directions. Animal studies have shown that hMT+/V5 contains motion direction-selective columns with specific directions organized side by side with their respective opposing motion direction counterparts (Albright et al., 1984; Geesaman et al., 1997; Diogo et al., 2003; Born and Bradley, 2005), an organization also probed using fMRI in humans (Zimmermann et al., 2011; but see below for alternative accounts). The observed difference for within-axis versus between-axis direction classification may potentially stem from such underlying cortical columnar organization (Kamitani and Tong, 2005; Haynes and Rees, 2006; Bartels et al., 2008). Alternatively, it has been suggested that classifying orientation preference reflects a much larger-scale (e.g., retinotopy) rather than columnar organization (Op de Beeck, 2010; Freeman et al., 2011, 2013). Interestingly, high-field fMRI studies showed that the fMRI signal carries information related to both large-scale and fine-scale (columnar level) biases (Gardumi et al., 2016; Pratte et al., 2016; Sengupta et al., 2017). The present study sheds important new light on the coding mechanism of motion direction within the hPT and demonstrates that the fMRI signal in the hPT contains directionspecific information and points toward an axis-of-motion organization. This result highlights intriguing similarities between the visual and auditory systems, arguing for common neural-coding strategies of motion directions across the senses. However, further studies are needed to test the similarities between the coding mechanisms implemented in visual and auditory motion-selective regions, and, more particularly, what drives the directional information captured with fMRI in the auditory cortex.

Supporting univariate motion selectivity results in bilateral hPT, MVPA revealed higher classification accuracies for moving sounds than for static sounds (Fig. $3 A, B$ ). However, despite minimal univariate activity elicited by sound source location in hPT, and the absence of reliable univariate differences in the activity elicited by each position (Fig. 2C), within-axis MVP classification results showed that sound source location information can be reliably decoded bilaterally in hPT (Fig. $3 C$ ). Our results are in line with those of previous studies showing that posterior regions in auditory cortex exhibit location sensitivity both in animals (Recanzone, 2000; Tian et al., 2001; Stecker et al., 2005) and humans (Alain et al., 2001; Zatorre et al., 2002; Warren and Griffiths, 2003; Brunetti et al., 2005; Krumbholz et al., 2005; Ahveninen et al., 2006, 2013; Deouell et al., 2007; Derey et al., 2016; Ortiz-Rios et al., 2017). In contrast to what was observed for motion direction, however, within-axis and across-axis classifications did not differ in hPT for static sounds. This indicates that sound source locations might not follow topographic representations that are similar to the one observed for auditory motion directions.

The extent to which the neural representations of motion directions and sound source locations overlap has long been debated (Grantham, 1986; Kaas et al., 1999; Romanski et al., 2000; Zatorre and Belin, 2001; Smith et al., 2004, 2007; Poirier et al., 2017). A number of neurophysiological studies have reported direction-selective neurons along the auditory pathway (Ahissar et al., 1992; Stumpf et al., 1992; Toronchuk et al., 1992; Spitzer and Semple, 1993; Doan et al., 1999; McAlpine et al., 2000). However, whether these neurons code similarly for auditory motion and sound source location remains debated (Poirier et al., 1997; McAlpine et al., 2000; Ingham et al., 2001; Oliver et al., 2003). In our study, cross-condition classification revealed that auditory motion (e.g., going to the left) and sound source location (being on the left) share partial neural representations in hPT (Fig. 3A). This observation suggests that there is a shared level of computation between sounds located on a given position and sounds 
moving toward this position. Low-level features of these two types of auditory stimuli vary in many ways and produce large differences at the univariate level in the cortex (Fig. 2B). However, perceiving, for instance, a sound going toward the left side or located on the left side evokes a sensation of location/direction in the external space that is common across conditions. Our significant cross-condition classification may therefore relate to the evoked sensation/perception of an object being/going to a common external spatial location. Electrophysiological studies in animals demonstrated that motion-selective neurons in the auditory cortex display a response profile that is similar for sounds located or moving toward the same position in external space (Ahissar et al., 1992; Poirier et al., 1997; Doan et al., 1999). Results from human psychophysiological and auditory evoked potential studies also strengthen the notion that sound source location contributes to motion perception (Strybel and Neale, 1994; Getzmann and Lewald, 2011). Our cross-condition MVPA results therefore extend the notion that motion directions and sound source locations might have common features that are partially shared for spatial hearing.

Significant cross-condition classification based on a multivariate fMRI signal has typically been considered as a demonstration that the region implements a common and abstracted representation of the tested conditions (Hong et al., 2012; Fairhall and Caramazza, 2013; Higgins et al., 2017). For instance, a recent study elegantly demonstrated that the human auditory cortex at least partly integrates interaural time differences (ITDs) and interaural level differences (ILDs) into a higher-order representation of auditory space based on significant cross-cue classification (training on ITD and classifying ILD, and the reverse; Higgins et al., 2017). In the present study, we argue that even if cross-condition MVP classification indeed demonstrates the presence of partially shared information across sound source location and direction, successful cross-MVPA results should, however, not be taken as evidence that the region implements a purely abstract representation of auditory space. Our successful across-condition classification (Fig. 4A) demonstrated that, even though there are shared representations for moving and static sounds within hPT, classifiers are able to easily distinguish motion directions from sound source locations (e.g., leftward vs left location). RSAs further supported the idea that moving and static sounds elicit distinct patterns in hPT (Fig. 4B-D). Altogether, our results suggest that hPT contains both motion direction and sound source location information but that the neural patterns related to these two conditions are only partially overlapping. Our observation of significant cross-condition classifications based on highly distinct patterns of activity between static and moving sounds may support the notion that even if location information could serve as a substrate for movement detection, motion encoding does not solely rely on location information (Ducommun et al., 2002; Getzmann, 2011; Poirier et al., 2017).

\section{References}

Ahissar M, Ahissar E, Bergman H, Vaadia E (1992) Encoding of soundsource location and movement: activity of single neurons and interactions between adjacent neurons in the monkey auditory cortex. J Neurophysiol 67:203-215.

Ahveninen J, Jaaskelainen IP, Raij T, Bonmassar G, Devore S, Hämäläinen M, Levänen S, Lin FH, Sams M, Shinn-Cunningham BG, Witzel T, Belliveau JW (2006) Task-modulated "what" and "where" pathways in human auditory cortex. Proc Natl Acad Sci U S A 103:14608-14613.

Ahveninen J, Huang S, Nummenmaa A, Belliveau JW, Hung AY, Jääskeläinen IP, Rauschecker JP, Rossi S, Tiitinen H, Raij T (2013) Evidence for distinct human auditory cortex regions for sound location versus identity processing. Nat Commun 4:2585.
Alain C, Arnott SR, Hevenor S, Graham S, Grady CL (2001) "What" and "where" in the human auditory system. Proc Natl Acad Sci U S A 98:12301-12306.

Albright TD, Desimone R, Gross CG (1984) Columnar organization of directionally selective cells in visual area MT of the macaque. J Neurophysiol 51:16-31.

Altman JA (1968) Are there neurons detecting direction of sound source motion? Exp Neurol 22:13-25.

Altman JA (1994) Processing of information concerning moving sound sources in the auditory centers and its utilization by brain integrative structures. Sens Syst 8:255-261.

Arnott SR, Binns MA, Grady CL, Alain C (2004) Assessing the auditory dual-pathway model in humans. Neuroimage 22:401-408.

Barrett DJ, Hall DA (2006) Response preferences for "what" and "where" in human non-primary auditory cortex. Neuroimage 32:968-977.

Bartels A, Logothetis NK, Moutoussis K (2008) fMRI and its interpretations: an illustration on directional selectivity in area V5/MT. Trends Neurosci 31:444-453.

Battal C, Rezk M, Mattioni S, Vadlamudi J, Collignon O (2018) Representation of auditory motion directions and sound source locations in the human planum temporale. bioRxiv. Advance online publication. Retrieved January 16, 2019. doi:10.1101/302497.

Baumgart F, Gaschler-Markefski B, Woldorff MG, Heinze HJ, Scheich H (1999) A movement-sensitive area in auditory cortex. Nature 400:724726.

Benjamini Y, Yekutieli D (2001) The control of the false discovery rate in multiple testing under dependency. Ann Stat 29:1165-1188.

Benson DA, Hienz RD, Goldstein MH Jr (1981) Single-unit activity in the auditory cortex of monkeys actively localizing sound sources: spatial tuning and behavioral dependency. Brain Res 219:249-267.

Blauert J (1982) Binaural localization. Scand Audiol Suppl 15:7-26.

Born RT, Bradley DC (2005) Structure and function of visual area MT. Annu Rev Neurosci 28:157-189.

Boudreau JC, Tsuchitani C (1968) Binaural interaction in the cat superior olive S segment. J Neurophysiol 31:442-454.

Braddick OJ, O'Brien JM, Wattam-Bell J, Atkinson J, Hartley T, Turner R (2001) Brain areas sensitive to coherent visual motion. Perception 30:6172.

Bremmer F, Schlack A, Shah NJ, Zafiris O, Kubischik M, Hoffmann K, Zilles K, Fink GR (2001) Polymodal motion processing in posterior parietal and premotor cortex: a human fMRI study strongly implies equivalencies between humans and monkeys. Neuron 29:287-296.

Brunetti M, Belardinelli P, Caulo M, Del Gratta C, Della Penna S, Ferretti, Ferretti A, Lucci G, Moretti A, Pizzella V, Tartaro A, Torquati K, Olivetti Belardinelli M, Romani GL (2005) Human brain activation during passive listening to sounds from different locations: an fMRI and MEG study. Hum Brain Mapp 26:251-261.

Carlile S, Leung J (2016) The perception of auditory motion. Trends Hear 20:2331216516644254.

Chang C, Lin C (2011) LIBSVM: a library for support vector machines. ACM Trans Intell Syst Technol 2:1-27.

Collignon O, Vandewalle G, Voss P, Albouy G, Charbonneau G, Lassonde M, Lepore F (2011) Functional specialization for auditory-spatial processing in the occipital cortex of congenitally blind humans. Proc Natl Acad Sci U S A 108:4435-4440.

Cox DD, Savoy RL (2003) Functional magnetic resonance imaging (fMRI) "brain reading": detecting and classifying distributed patterns of fMRI activity in human visual cortex. Neuroimage 19:261-270.

De Martino F, Valente G, Staeren N, Ashburner J, Goebel R, Formisano E (2008) Combining multivariate voxel selection and support vector machines for mapping and classification of fMRI spatial patterns. Neuroimage 43:44-58.

Deouell LY, Heller AS, Malach R, D’Esposito MD, Knight RT (2007) Cerebral responses to change in spatial location of unattended sounds. Neuron 55:985-996.

Derey K, Valente G, de Gelder B, Formisano E (2016) Opponent coding of sound location (azimuth) in planum temporale is robust to sound-level variations. Cereb Cortex 26:450-464.

Destrieux C, Fischl B, Dale A, Halgren E (2010) Automatic parcellation of human cortical gyri and sulci using standard anatomical nomenclature. Neuroimage 53:1-15.

Diogo AC, Soares JG, Koulakov A, Albright TD, Gattass R (2003) Electro- 
physiological imaging of functional architecture in the cortical middle temporal visual area of cebus apella monkey. J Neurosci 23:3881-3898.

Doan DE, Saunders JC, Field F, Ingham NJ, Hart HC, McAlpine D (1999) Sensitivity to simulated directional sound motion in the rat primary auditory cortex. J Neurophysiol 81:2075-2087.

Dormal G, Rezk M, Yakobov E, Lepore F, Collignon O (2016) Auditory motion in the sighted and blind: early visual deprivation triggers a largescale imbalance between auditory and "visual" brain regions. Neuroimage 134:630-644.

Ducommun CY, Murray MM, Thut G, Bellmann A, Viaud-Delmon I, Clarke S, Michel CM (2002) Segregated processing of auditory motion and auditory location: an ERP mapping study. Neuroimage 16:76-88.

Edelman S, Grill-Spector K, Kushnir T, Malach R (1998) Toward direct visualization of the internal shape represetation space by fMRI. Psychobiology 26:309-321.

Eickhoff SB, Paus T, Caspers S, Grosbras MH, Evans AC, Zilles K, Amunts K (2007) Assignment of functional activations to probabilistic cytoarchitectonic areas revisited. Neuroimage 36:511-521.

Fairhall SL, Caramazza A (2013) Brain regions that represent amodal conceptual knowledge. J Neurosci 33:10552-10558.

Freeman J, Brouwer GJ, Heeger DJ, Merriam EP (2011) Orientation decoding depends on maps, not columns. J Neurosci 31:4792-4804.

Freeman J, Heeger DJ, Merriam EP (2013) Coarse-scale biases for spirals and orientation in human visual cortex. J Neurosci 33:19695-19703.

Gardumi A, Ivanov D, Hausfeld L, Valente G, Formisano E, Uludağ, K, Uludağ K (2016) The effect of spatial resolution on decoding accuracy in fMRI multivariate pattern analysis. Neuroimage 132:32-42.

Geesaman BJ, Born RT, Andersen RA, Tootell RB (1997) Maps of complex motion selectivity in the superior temporal cortex of the alert macaque monkey: a double-label 2-deoxyglucose study. Cereb Cortex 7:749-757.

Getzmann S (2011) Auditory motion perception: onset position and motion direction are encoded in discrete processing stages. Eur J Neurosci 33:1339-1350.

Getzmann S, Lewald J (2011) The effect of spatial adaptation on auditory motion processing. Hear Res 272:21-29.

Getzmann S, Lewald J (2012) Cortical processing of change in sound location: smooth motion versus discontinuous displacement. Brain Res 1466: 119-127.

Goldberg JM, Brown PB (1969) Response of binaural neurons of dog superior olivary complex to dichotic tonal stimuli: some physiological mechanisms of sound localization. J Neurophysiol 32:613-636.

Goodale MA, Milner AD (1992) Separate visual pathways for perception and action. Trends Neurosci 15:20-25.

Grantham DW (1986) Detection and discrimination of simulated motion of auditory targets in the horizontal plane. J Acoust Soc Am 79:19391949.

Grothe B, Pecka M, McAlpine D (2010) Mechanisms of sound localization in mammals. Physiol Rev 90:983-1012.

Hall DA, Moore DR (2003) Auditory neuroscience: the salience of looming sounds. Curr Biol 13:R91-R93.

Hall DA, Haggard MP, Akeroyd MA, Palmer AR, Summerfield AQ, Elliott MR, Gurney EM, Bowtell RW (1999) "Sparse" temporal sampling in auditory fMRI. Hum Brain Mapp 7:213-223.

Haxby JV, Gobbini MI, Furey ML, Ishai A, Schouten JL, Pietrini P (2001) Distributed and overlapping representations of faces and objects in ventral temporal cortex. Science 293:2425-2430.

Haynes JD, Rees G (2006) Decoding mental states from brain activity in humans. Nat Rev Neurosci 7:523-534.

Higgins NC, McLaughlin SA, Rinne T, Stecker GC (2017) Evidence for cueindependent spatial representation in the human auditory cortex during active listening. Proc Natl Acad Sci U S A 114:E7602-E7611.

Hong SW, Tong F, Seiffert AE (2012) Direction-selective patterns of activity in human visual cortex suggest common neural substrates for different types of motion. Neuropsychologia 50:514-521.

Imig TJ, Irons WA, Samson FR (1990) Single-unit selectivity to azimuthal direction and sound pressure level of noise bursts in cat high-frequency primary auditory cortex. J Neurophysiol 63:1448-1466.

Ingham NJ, Hart HC, McAlpine D (2001) Spatial receptive fields of inferior colliculus neurons to auditory apparent motion in free field. J Neurophysiol 85:23-33.

Kaas JH, Hackett TA, Tramo MJ (1999) Auditory processing in primate cerebral cortex. Curr Opin Neurobiol 9:164-170.
Kamitani Y, Tong F (2005) Decoding the visual and subjective contents of the human brain. 8:679-685.

Kamitani Y, Tong F (2006) Decoding seen and attended motion directions from activity in the human visual cortex. Curr Biol 16:1096-1102.

Knudsen EI, Konishi M (1978) A neural map of auditory space in the owl. Science 200:795-797.

Kriegeskorte N, Formisano E, Sorger B, Goebel R (2007) Individual faces elicit distinct response patterns in human anterior temporal cortex. Proc Natl Acad Sci U S A 104:20600-20605.

Kriegeskorte N, Mur M, Bandettini P (2008) Representational similarity analysis-connecting the branches of systems neuroscience. Front Syst Neurosci 2:4.

Krumbholz K, Schönwiesner M, Rübsamen R, Zilles K, Fink GR, von Cramon DY (2005) Hierarchical processing of sound location and motion in the human brainstem and planum temporale. Eur J Neurosci 21:230-238.

Lakatos S, Shepard RN (1997) Contraints common to apparent motion in visual, tactile, and auditory space. J Exp Psychol Hum Percept Perform 23:1050-1060.

Lewis JW, Beauchamp MS, DeYoe EA (2000) A comparison of visual and auditory motion processing in human cerebral cortex. Cereb Cortex 10:873-888.

Lomber SG, Malhotra S (2008) Double dissociation of "what" and "where" processing in auditory cortex. Nat Neurosci 11:609-616.

Maeder PP, Meuli RA, Adriani M, Bellmann A, Fornari E, Thiran JP, Pittet A, Clarke S (2001) Distinct pathways involved in sound recognition and localization: a human fMRI study. Neuroimage 14:802-816.

McAlpine D, Jiang D, Shackleton TM, Palmer AR (2000) Responses of neurons in the inferior colliculus to dynamic interaural phase cues: evidence for a mechanism of binaural adaptation. J Neurophysiol 83:1356-1365.

Middlebrooks J, Pettigrew JD (1981) Functional classes of neurons in primary auditory cortex of the cat distinguished by sensitivity to sound location. J Neurosci 1:107-120.

Middlebrooks JC, Green DM (1991) Sound localization by human listeners. Annu Rev Psychol 42:135-159.

Movshon JA, Newsome WT (1996) Visual response properties of striate cortical neurons projecting to area MT in macaque monkeys. J Neurosci 16:7733-7741.

Newsome WT, Paré EB (1988) A selective impairment of motion perception following lesions of the middle temporal visual area (MT). J Neurosci 8:2201-2211.

Oliver DL, Beckius GE, Bishop DC, Loftus WC, Batra R (2003) Topography of interaural temporal disparity coding in projections of medial superior olive to inferior colliculus. J Neurosci 23:7438-7449.

Oosterhof NN, Connolly AC, Haxby JV (2016) CoSMoMVPA: multimodal multivariate pattern analysis of neuroimaging data in Matlab/ GNU octave. Front Neuroinform 10:27.

Op de Beeck HP (2010) Against hyperacuity in brain reading: spatial smoothing does not hurt multivariate fMRI analyses? Neuroimage 49: 1943-1948.

Ortiz-Rios M, Azevedo FAC, Kuśmierek P, Balla DZ, Munk MH, Keliris GA, Logothetis NK, Rauschecker JP (2017) Widespread and opponent fMRI signals represent sound location in macaque auditory cortex. Neuron 93:971-983.e4.

Pavani F, Macaluso E, Warren JD, Driver J, Griffiths TD (2002) A common cortical substrate activated by horizontal and vertical sound movement in the human brain. Curr Biol 12:1584-1590.

Poirier C, Collignon O, Devolder AG, Renier L, Vanlierde A, Tranduy D, Scheiber C (2005) Specific activation of the V5 brain area by auditory motion processing: an fMRI study. Brain Res Cogn Brain Res 25:650658.

Poirier C, Baumann S, Dheerendra P, Joly O, Hunter D, Balezeau F, Sun L, Rees A, Petkov CI, Thiele A, Griffiths TD (2017) Auditory motionspecific mechanisms in the primate brain. PLoS Biol 15:e2001379.

Poirier P, Jiang H, Lepore F, Guillemot JP (1997) Positional, directional and speed selectivities in the primary auditory cortex of the cat. Hear Res 113:1-13.

Pratte MS, Sy JL, Swisher JD, Tong F (2016) Radial bias is not necessary for orientation decoding. Neuroimage 127:23-33.

Rajan R, Aitkin LM, Irvine DR (1990) Azimuthal sensitivity of neurons in primary auditory cortex of cats. II. Organization along frequency-band strips. J Neurophysiol 64:888-902.

Rauschecker JP, Tian B (2000) Mechanisms and streams for processing of 
"what" and "where" in auditory cortex. Proc Natl Acad Sci U S A 97:11800-11806.

Recanzone GH (2000) Spatial processing in the auditory cortex of the macaque monkey. Proc Natl Acad Sci U S A 97:11829-11835.

Romanski LM, Tian B, Fritz J, Mishkin M, Goldman-Rakic PS, Rauschecker JP (1999) Dual streams of auditory afferents target multiple domains in the primate prefrontal cortex. Nat Neurosci 2:1131-1136.

Romanski LM, Tian B, Fritz JB, Mishkin M, Goldman-Rakic PS, Rauschecker JP (2000) Reply to "What", "where" and "how" in auditory cortex. Nat Neurosci 3:966.

Searle CL, Braida LD, Davis MF, Colburn HS (1976) Model for auditory localization. J Acoust Soc Am 60:1164-1175.

Sengupta A, Yakupov R, Speck O, Pollmann S, Hanke M (2017) The effect of acquisition resolution on orientation decoding from V1 BOLD fMRI at 7 T. Neuroimage 148:64-76.

Smith KR, Okada K, Saberi K, Hickok G (2004) Human cortical auditory motion areas are not motion selective. Neuroreport 15:1523-1526.

Smith KR, Saberi K, Hickok G (2007) An event-related fMRI study of auditory motion perception: no evidence for a specialized cortical system. Brain Res 1150:94-99.

Smith KR, Hsieh IH, Saberi K, Hickok G (2010) Auditory spatial and object processing in the human planum temporale: no evidence for selectivity. J Cogn Neurosci 22:632-639.

Spitzer MW, Semple MN (1993) Responses of inferior colliculus neurons to time-varying interaural phase disparity: effects of shifting the locus of virtual motion. J Neurophysiol 69:1245-1263.

Stecker GC, Harrington IA, Middlebrooks JC (2005) Location coding by opponent neural populations in the auditory cortex. PLoS Biol 3:e78.

Stelzer J, Chen Y, Turner R (2013) Statistical inference and multiple testing correction in classification-based multi-voxel pattern analysis (MVPA): random permutations and cluster size control. Neuroimage 65:69-82.

Strybel TZ, Neale W (1994) The effect of burst duration, interstimulus onset interval, and loudspeaker arrangement on auditory apparent motion in the free field. J Acoust Soc Am 96:3463-3475.

Stumpf E, Toronchuk JM, Cynader MS (1992) Neurons in cat primary auditory cortex sensitive to correlates of auditory motion in threedimensional space. Exp Brain Res 88:158-168.
Tian B, Reser D, Durham A, Kustov A, Rauschecker JP (2001) Functional specialization in rhesus monkey auditory cortex. Science 292:290-293.

Tootell RB, Reppas JB, Dale AM, Look RB, Sereno MI, Malach R, Brady TJ, Rosen BR (1995) Visual motion aftereffect in human cortical area MT revealed by functional magnetic resonance imaging. Nature 375:139-141.

Toronchuk JM, Stumpf E, Cynader MS (1992) Auditory cortex neurons sensitive to correlates of auditory motion: underlying mechanisms. Exp Brain Res 88:169-180.

Ungerleider LG, Mishkin M (1982) Two cortical visual systems. In: Analysis of visual behavior (Ingle DJ, ed), pp 549-586. Cambridge, MA: MIT.

Warren JD, Griffiths TD (2003) Distinct mechanisms for processing spatial sequences and pitch sequences in the human auditory brain. J Neurosci 23:5799-5804.

Warren J, Zielinski B, Green GG, Rauschecker JP, Griffiths TD (2002) Perception of sound-source motion by the human brain. Neuron 34:139148.

Watson JD, Myers R, Frackowiak RS, Hajnal JV, Woods RP, Mazziotta JC, Shipp S, Zeki S (1993) Area V5 of the human brain: evidence from a combined study using positron emission tomography and magnetic resonance imaging. Cereb Cortex 3:79-94.

Yarkoni T, Poldrack RA, Nichols TE, Van Essen DC, Wager TD (2011) Large-scale automated synthesis of human functional neuroimaging data. Nat Methods 8:665-670.

Zabicki A, de Haas B, Zentgraf K, Stark R, Munzert J, Krüger B (2017) Imagined and executed actions in the human motor system: testing neural similarity between execution and imagery of actions with a multivariate approach. Cereb Cortex 27:4523-4536.

Zatorre RJ, Belin P (2001) Spectral and temporal processing in human auditory cortex. Cereb Cortex 11:946-953.

Zatorre RJ, Bouffard M, Ahad P, Belin P (2002) Where is "where" in the human auditory cortex? Nat Neurosci 5:905-909.

Zeng H, Constable RT (2002) Image distortion correction in EPI: comparison of field mapping with point spread function mapping. Magn Reson Med 48:137-146.

Zimmermann J, Goebel R, de Martino F, van de Moortele PF, Feinberg D, Adriany G, Chaimow D, Shmuel A, Uğurbil K, Yacoub E (2011) Mapping the organization of axis of motion selective features in human area MT using high-field fMRI. PLoS One 6:e28716. 